TRANSACTIONS OF THE

AMERICAN MATHEMATICAL SOCIETY

Volume 355, Number 10, October 2003, Pages 4171-4186

S 0002-9947(03)03320-8

Article electronically published on June 10, 2003

\title{
GENERALIZED ASSOCIAHEDRA VIA QUIVER REPRESENTATIONS
}

\author{
BETHANY MARSH, MARKUS REINEKE, AND ANDREI ZELEVINSKY
}

\begin{abstract}
We provide a quiver-theoretic interpretation of certain smooth complete simplicial fans associated to arbitrary finite root systems in recent work of S. Fomin and A. Zelevinsky. The main properties of these fans then become easy consequences of the known facts about tilting modules due to K. Bongartz, D. Happel and C. M. Ringel.
\end{abstract}

\section{INTRODUCTION}

The goal of this paper is to provide a conceptual interpretation for generalized associahedra introduced in [7]. Recall that in [7, Section 3], to every finite (crystallographic) root system $\Phi$ is associated a smooth complete simplicial fan $\Delta(\Phi)$ in the ambient real vector space. (Here smoothness means that every full-dimensional cone of $\Delta(\Phi)$ is generated by a $\mathbb{Z}$-basis of the root lattice $Q$, and completeness means that the union of all cones is the whole ambient space.) As proved in [5], $\Delta(\Phi)$ is the normal fan of a simple convex polytope. With some abuse of terminology, both $\Delta(\Phi)$ and the corresponding simple polytope are referred to as the generalized associahedron associated to $\Phi$. This construction includes as special cases the well-known associahedron (or Stasheff polytope) [13] for $\Phi$ of type $A$, and the Bott-Taubes cyclohedron [4] for $\Phi$ of type $B$ or $C$.

The construction in [7, Section 3] appears somewhat ad hoc (as indicated in [7], it is motivated by the developing theory of cluster algebras [6], but this connection is still unpublished). In this paper, we present a natural interpretation of generalized associahedra in terms of quiver representations. With this interpretation, the main results in [7, Section 3] become easy consequences of the known facts about tilting representations [8].

To explain our results in more detail, let us recall the main ingredients of the construction in [7]:

(1) The set of almost positive roots $\Phi_{\geq-1}=\Phi_{>0} \cup(-\Pi)$, where $\Pi=\left\{\alpha_{i}\right.$ : $i \in I\}$ is a fixed set of simple roots in the root system $\Phi$, and $\Phi_{>0}$ is the corresponding set of positive roots. Almost positive roots are precisely the generators of one-dimensional cones in the fan $\Delta(\Phi)$.

Received by the editors May 26, 2002 and, in revised form, February 8, 2003.

2000 Mathematics Subject Classification. Primary 52B11, 16G20, 17B20; Secondary 17B37, $05 \mathrm{E} 99$.

Andrei Zelevinsky's research was supported in part by NSF grants \#DMS-9971362 and DMS0200299. Bethany Marsh's research was supported in part by EPSRC grant GR/R17546/01. All authors were supported in part by a University of Leicester Research Fund Grant. 
(2) The function $\Phi_{\geq-1} \times \Phi_{\geq-1} \rightarrow \mathbb{Z}_{\geq 0}$ assigning to each $\alpha, \beta \in \Phi_{\geq-1}$ their compatibility degree $(\alpha \| \bar{\beta})$. A subset $C \subset \Phi_{\geq-1}$ generates a cone in $\Delta(\Phi)$ if and only if it is compatible, i.e., $(\alpha \| \beta)=0$ for any $\alpha, \beta \in C$.

(3) The group of piecewise-linear transformations of the root lattice $Q$ generated by "truncated simple reflections" $\sigma_{i}$ for $i \in I$. Every $\sigma_{i}$ preserves $\Phi_{\geq-1}$.

(4) In the case when $\Phi$ is irreducible, the group generated by the $\sigma_{i}$ contains two distinguished involutions $\tau_{+}$and $\tau_{-}$that preserve $\Delta(\Phi)$.

Our aim is to provide natural interpretations of all these notions in terms of quiver representations. For simplicity, we only work with simply-laced root systems, i.e., those corresponding to Dynkin diagrams of the $A D E$-type. (The general case reduces to this with the help of a familiar "folding" procedure; see, e.g., [10, Chapter 14], or by modifying our constructions in the spirit of [9], [14].) Let $\Gamma$ be a quiver (= oriented graph) whose underlying graph is a disjoint union of Dynkin diagrams of types $A_{n}, D_{n}, E_{6}, E_{7}, E_{8}$. By Gabriel's theorem, the dimension vector map identifies isomorphism classes of indecomposable representations of $\Gamma$ with positive roots of the corresponding root system $\Phi$. As a first step in our program, we include the category of representations of $\Gamma$ into a bigger category of decorated representations (see Section 2 below). A suitable modification of the dimension vector map establishes a bijection $\alpha \mapsto U_{\alpha}^{\Gamma}$ between almost positive roots in $\Phi$ and isomorphism classes of indecomposable decorated representations of $\Gamma$.

As a next step, we find a natural interpretation for the compatibility degree function. To do this, in Section 3 we construct a bifunctor $E_{\Gamma}$ assigning a finitedimensional vector space to every two decorated representations of $\Gamma$. We then define the $\Gamma$-compatibility degree function $\Phi_{\geq-1} \times \Phi_{\geq-1} \rightarrow \mathbb{Z}_{\geq 0}$ by setting $(\alpha \| \beta)_{\Gamma}=$ $\operatorname{dim} E_{\Gamma}\left(U_{\alpha}^{\Gamma}, U_{\beta}^{\Gamma}\right)$. As in (2) above, we call a subset $C \subset \Phi_{\geq-1} \Gamma$-compatible if $(\alpha \| \beta)_{\Gamma}=0$ for any $\alpha, \beta \in C$. We prove that the cones generated by $\Gamma$-compatible subsets form a smooth complete simplicial fan in the ambient space $Q_{\mathbb{R}}$ (Theorem 3.7). This result follows without much effort from known results about tilting representations due to D. Happel and C. M. Ringel [8] and K. Bongartz [2].

The original compatibility degree and the corresponding fan $\Delta(\Phi)$ appear as a special case of this construction corresponding to an alternating quiver $\Gamma_{0}$ for which every vertex is a source or a sink. Our verification of this fact involves the final step in our program: finding a quiver-theoretic interpretation of the piecewise-linear involutions $\sigma_{i}$ acting on the root lattice $Q$ and preserving $\Phi_{\geq-1}$. We do this by extending the familiar reflection functors $S_{i}$ (see, e.g., [1]) to the functors $\Sigma_{i}$ on the category of decorated representations; more precisely, each $\Sigma_{i}$ sends decorated representations of a quiver $\Gamma$ to those of the quiver $s_{i} \Gamma$ obtained from $\Gamma$ by reversing all arrows at a vertex $i$ that is a source or a sink in $\Gamma$. The main advantage of the extended reflection functors is that each $\Sigma_{i}$ induces a bijection between isomorphism classes of decorated representations of $\Gamma$ and those of $s_{i} \Gamma$ (recall that the classical reflection functors $S_{i}$ send some nonzero quiver representations to zero). Furthermore, $\Sigma_{i}^{2}(M)$ is isomorphic to $M$ for every decorated representation $M$. Note, however, that $\Sigma_{i}$ is not an equivalence of categories; in fact, it sends some nonzero morphisms to zero.

The key property of the functors $\Sigma_{i}$ for our purposes is that they are compatible with the bifunctors $E_{\Gamma}$ in the following sense: the vector space $E_{\Gamma}(M, N)$ is isomorphic to $E_{s_{i} \Gamma}\left(\Sigma_{i} M, \Sigma_{i} N\right)$ for every two decorated representations $M$ and $N$ of 
$\Gamma$ (Theorem 4.7 below). Returning to piecewise-linear involutions $\sigma_{i}$, we show that each $\sigma_{i}$ is obtained by combining the reflection functor $\Sigma_{i}$ with the signed dimension vector map for decorated quiver representations (see Lemma 4.5 for a precise statement). This implies the following important property of the $\Gamma$-compatibility degree: $\left(\sigma_{i} \alpha \| \sigma_{i} \beta\right)_{s_{i} \Gamma}=(\alpha \| \beta)_{\Gamma}$ (Proposition 4.10).

The role of two distinguished piecewise-linear involutions $\tau_{+}$and $\tau_{-}$also becomes clear in this context. The functors $\Sigma_{i}$ give rise to a groupoid whose objects correspond to different orientations $\Gamma$ of a fixed Dynkin graph, and each $\Sigma_{i}$ is viewed as a morphism from $\Gamma$ to $s_{i} \Gamma$. It is natural to enlarge this groupoid by the duality functor $D$ that sends every quiver $\Gamma$ to the opposite quiver obtained from $\Gamma$ by reversing all arrows. We study these two groupoids in Section 5 (in a more general setting where the Dynkin graph is replaced by an arbitrary finite tree). We prove, in particular, that for the groupoid generated by the $\Sigma_{i}$ and $D$, the automorphism group of an alternating quiver $\Gamma_{0}$ is generated by two involutions $T_{+}$and $T_{-}$, whose induced action on the root lattice $Q$ is given by the involutions $\tau_{+}$and $\tau_{-}$from [7].

We conclude the paper by an amusing application of our results to the theory of ordinary quiver representations. We say that a set $C$ of isomorphism classes of indecomposable representations of a quiver $\Gamma$ is Ext-free (also called a partial tilting set) if $\operatorname{Ext}^{1}(M, N)=0$ for every $M, N \in C$. The Ext-free sets form a simplicial complex, and we show that the $f$-vector of this complex (i.e., the number of Ext-free sets of any given size) is an invariant of the underlying Dynkin graph, i.e., is independent of the choice of the orientation $\Gamma$ (Proposition 6.1). Note that the complexes of Ext-free sets corresponding to different orientations of the same Dynkin graph are in general not isomorphic. Passing from ordinary quiver representations to decorated ones allows us to view the complex of Ext-free sets for $\Gamma$ as a subcomplex of the complex of $\Gamma$-compatible sets discussed above; these bigger complexes are all isomorphic to each other, by the action of extended reflection functors $\Sigma_{i}$.

\section{Decorated Quiver Representations}

Let $\Gamma$ be a Dynkin quiver (that is, the underlying unoriented graph is a disjoint union of Dynkin diagrams of types $A_{n}, D_{n}, E_{6}, E_{7}, E_{8}$ ), and let $I$ be the set of vertices. Fix once and for all an algebraically closed field $k$. Let rep $\Gamma$ be the category of finite-dimensional $k$-representations of $\Gamma$; the objects $M$ of rep $\Gamma$ thus consist of tuples of $k$-vector spaces $\left(M_{i}\right)_{i \in I}$ and tuples of linear maps $\left(M_{a}: M_{i} \rightarrow M_{j}\right)_{a: i \rightarrow j}$ parametrized by the arrows $a$ in $\Gamma$. For each vertex $i \in I$, denote by $E_{i}$ the associated simple representation. The space of homomorphisms (resp. extensions) between representations $M, N \in \operatorname{rep} \Gamma$ is denoted by $\operatorname{Hom}_{\Gamma}(M, N)$ (resp. $\left.\operatorname{Ext}_{\Gamma}^{1}(M, N)\right)$. The $k$-linear dual of a $k$-vector space $X$ is denoted by $X^{*}$.

Let $\Phi$ be the root system associated with the Dynkin graph underlying $\Gamma$, let $\Phi_{>0}$ be the positive roots, and let $\Phi_{\geq-1}$ be the union of $\Phi_{>0}$ and the negative simple roots $-\Pi=\left\{-\alpha_{i}: i \in I\right\}$. For a subset $J \subset I$, let $\Phi(J)$ be the root subsystem of roots whose support is contained in $J$.

Let $Q$ be the root lattice, and let $Q_{+}$be the positive span of $\Phi_{>0}$ in $Q$. For $\gamma \in Q$ and $i \in I$, denote by $\left[\gamma: \alpha_{i}\right]$ the multiplicity of $\alpha_{i}$ in $\gamma$.

The Grothendieck group of the category rep $\Gamma$ can be identified canonically with $Q$ via the dimension vector map $\underline{\operatorname{dim}}: K_{0}(\operatorname{rep} \Gamma) \rightarrow Q$ given on objects $M \in \operatorname{rep} \Gamma$ 
by

$$
\underline{\operatorname{dim}}(M)=\sum_{i \in I}\left(\operatorname{dim} M_{i}\right) \alpha_{i}
$$

Associate to $\Gamma$ a new quiver $\widetilde{\Gamma}=\Gamma \cup\left\{i^{-}: i \in I\right\}$, with no arrows pointing from or to the added vertices $i^{-}$. The category rep $\widetilde{\Gamma}$ will be called the decorated representation category for $\Gamma$. The simple representation $E_{i^{-}}$will be denoted by $E_{i}^{-}$; the objects of rep $\widetilde{\Gamma}$ are thus of the form $M=M^{+} \oplus V$, where $M^{+} \in \operatorname{rep} \Gamma$, and $V=\bigoplus_{i \in I}\left(E_{i}^{-} \otimes V_{i}\right)$ is viewed as an $I$-graded vector space. More explicitly, an object of rep $\widetilde{\Gamma}$ consists of tuples of vector spaces $M_{i}$ and $V_{i}$ for $i \in I$, together with linear maps $M_{a}: M_{i} \rightarrow M_{j}$ for all arrows $a: i \rightarrow j$ in $\Gamma$; morphisms between two such pairs of tuples $\left(M_{i}, V_{i}\right)$ and $\left(N_{i}, W_{i}\right)$ consist of linear maps $f_{i}: M_{i} \rightarrow N_{i}$, together with linear maps $\varphi_{i}: V_{i} \rightarrow W_{i}$ such that $f_{j} \circ M_{a}=N_{a} \circ f_{i}$ for all arrows $a: i \rightarrow j$.

The space of $I$-graded homomorphisms of $I$-graded vector spaces $V, W$ will be denoted by $\operatorname{Hom}^{I}(V, W)$.

The category rep $\Gamma$ is viewed as a subcategory of rep $\widetilde{\Gamma}$ in the obvious way. Define the signed dimension vector $\underline{\operatorname{sim}}(M)$ of $M=M^{+} \oplus V$ by

$$
\underline{\operatorname{sdim}}(M)=\underline{\operatorname{dim}} M^{+}-\sum_{i \in I}\left(\operatorname{dim} V_{i}\right) \alpha_{i}=\sum_{i \in I}\left(\operatorname{dim} M_{i}-\operatorname{dim} V_{i}\right) \alpha_{i} .
$$

By Gabriel's theorem, the dimension vector induces a bijection between the isoclasses of indecomposable representations in rep $\Gamma$ and the positive roots $\Phi_{>0}$.

We have the following extension of Gabriel's theorem, which is immediate from the definition of the decorated representation category:

Proposition 2.1. The map sdim induces a bijection $\alpha \mapsto\left[U_{\alpha}^{\Gamma}\right]$ between the roots $\alpha \in \Phi_{\geq-1}$ and isoclasses $\left[U_{\alpha}^{\Gamma}\right]$ of indecomposable objects in rep $\widetilde{\Gamma}$.

\section{Clusters from decorated Representations}

For decorated representations $M=M^{+} \oplus V$ and $N=N^{+} \oplus W$ in $\operatorname{rep} \widetilde{\Gamma}$, we define

$$
\begin{gathered}
E_{\Gamma}(M, N)=\operatorname{Ext}_{\Gamma}^{1}\left(M^{+}, N^{+}\right) \oplus \operatorname{Ext}_{\Gamma}^{1}\left(N^{+}, M^{+}\right)^{*} \\
\oplus \operatorname{Hom}^{I}\left(M^{+}, W\right) \oplus \operatorname{Hom}^{I}\left(V, N^{+}\right) .
\end{gathered}
$$

Obviously, this defines a bifunctor

$$
E_{\Gamma}\left({ }_{-}\right):(\operatorname{rep} \widetilde{\Gamma})^{\mathrm{op}} \times \operatorname{rep} \widetilde{\Gamma} \rightarrow \operatorname{vect},
$$

contravariant in the first argument, and covariant in the second one. Here vect denotes the category of finite-dimensional vector spaces.

In view of (3.1), we have the following characterization of decorated representations $M=M^{+} \oplus V$ such that $E_{\Gamma}(M, M)=0$ :

$$
\begin{aligned}
& E_{\Gamma}(M, M)=0 \text { if and only if } \operatorname{Ext}_{\Gamma}^{1}\left(M^{+}, M^{+}\right)=0, \\
& \text { and } M^{+} \text {and } V \text { have disjoint supports in } I .
\end{aligned}
$$

We define the $\Gamma$-compatibility degree of two roots $\alpha, \beta \in \Phi_{\geq-1}$ by

$$
(\alpha \| \beta)_{\Gamma}=\operatorname{dim} E_{\Gamma}\left(U_{\alpha}^{\Gamma}, U_{\beta}^{\Gamma}\right) .
$$


This definition makes it clear that $(\|)_{\Gamma}$ is symmetric and compatible with root subsystems in the following sense: if $\Gamma(J)$ denotes the full subquiver of $\Gamma$ supported on a subset of vertices $J \subset I$, and if $\alpha, \beta \in \Phi_{\geq-1}(J)$, then $(\alpha \| \beta)_{\Gamma(J)}=(\alpha \| \beta)_{\Gamma}$ (compare [7, Proposition 3.3]). There is an additional symmetry property involving the natural duality functor $D: \operatorname{rep} \widetilde{\Gamma} \rightarrow \operatorname{rep} \widetilde{\Gamma}^{\text {op }}$ that acts by replacing all vector spaces and maps by their duals, thus replacing $\Gamma$ with the opposite orientation $\Gamma^{\mathrm{op}}$ :

Proposition 3.1. For all roots $\alpha, \beta \in \Phi_{\geq-1}$, we have $(\alpha \| \beta)_{\Gamma}=(\alpha \| \beta)_{\Gamma^{\mathrm{op}}}$.

This follows at once from the natural identification

$$
E_{\Gamma \mathrm{op}}(D N, D M) \simeq E_{\Gamma}(M, N) .
$$

We can now define $\Gamma$-compatible subsets and $\Gamma$-clusters as in [7, Definition 3.4].

Definition 3.2. A subset $C$ of $\Phi_{\geq-1}$ is called $\Gamma$-compatible if $(\alpha \| \beta)_{\Gamma}=0$ for all $\alpha, \beta \in C$. The subset $C$ is called a $\Gamma$-cluster if it is a maximal (by inclusion) $\Gamma$-compatible subset.

Definition 3.3. The negative support $S(C)$ of a subset $C$ of $\Phi_{\leq-1}$ is defined by $S(C)=\left\{i \in I:-\alpha_{i} \in C\right\}$. The subset $C$ is called positive if $C \subset \Phi_{>0}$, i.e., $S(C)=\emptyset$.

Moreover, [7, Proposition 3.6] holds with the same proof.

Proposition 3.4. For every subset $J \subset I$, the correspondence $C \mapsto C-\left\{-\alpha_{i}\right.$ : $i \in J\}$ defines a bijection between $\Gamma$-compatible subsets (resp. clusters) for $\Phi$ with negative support $J$ and positive $\Gamma$-compatible subsets (resp. clusters) for $\Phi(I-J)$.

We can now prove the main properties of $\Gamma$-clusters.

Proposition 3.5 (Purity). All $\Gamma$-clusters have the same size $n$, the rank of the root system $\Phi$. Moreover, each $\Gamma$-cluster is a $\mathbb{Z}$-basis for the root lattice $Q$.

Proof. In view of Proposition 3.4, it suffices to prove our statements for positive $\Gamma$-clusters. To any subset $C \subset \Phi_{>0}$ let us associate a $\Gamma$-representation $X_{C}=$ $\bigoplus_{\alpha \in C} U_{\alpha}^{\Gamma}$. By our definitions, $C$ is $\Gamma$-compatible if and only if $\operatorname{Ext}^{1}\left(X_{C}, X_{C}\right)=0$. Now we use the theory of tilting modules introduced in [8]. In our situation, they can be defined as follows (see [8, Theorem 4.5]): a $\Gamma$-representation $T$ is tilting if $\operatorname{Ext}^{1}(T, T)=0$ and $T$ has precisely $n$ different isoclasses of indecomposable direct summands.

Now let $C$ be a positive $\Gamma$-cluster. By [2, Lemma 2.1], $X_{C}$ is a direct summand of a tilting module $T$. By the maximality property of $C$, the representation $X_{C}$ contains all isoclasses of indecomposable direct summands of $T$ and so $|C|=n$. The fact that $C$ is a $\mathbb{Z}$-basis for $Q$ is contained in the proof of [8, Lemma 4.3].

Proposition 3.6 (Cluster expansion). Each element $\gamma \in Q$ has a unique $\Gamma$-cluster expansion, that is, $\gamma=\sum_{\alpha \in \Phi_{\geq-1}} m_{\alpha} \alpha$ such that $m_{\alpha} \in \mathbb{Z}_{\geq 0}$ for all $\alpha$, and $m_{\alpha} m_{\beta}=$ 0 whenever $\alpha, \beta \in \Phi_{\geq-1}$ are not $\Gamma$-compatible.

Proof. We prove that for each $\gamma \in Q$, there exists a unique decorated representation $M=M^{+} \oplus V \in \operatorname{rep} \widetilde{\Gamma}$ such that $\underline{\operatorname{sim}} M=\gamma$ and $E_{\Gamma}(M, M)=0$ (the proposition follows from this by definition of the $\Gamma$-compatibility degree). In view of (3.2), the condition $E_{\Gamma}(M, M)=0$ means that $\operatorname{Ext}_{\Gamma}^{1}\left(M^{+}, M^{+}\right)=0$, and $M^{+}$and $V$ have disjoint supports in $I$. Denote by $d=\sum_{i \in I} d_{i} \alpha_{i}$ the dimension vector of $M^{+}$. 
Note that it is uniquely determined by $\gamma$, namely $d=\sum_{i \in I} \max \left(\left[\gamma: \alpha_{i}\right], 0\right) \alpha_{i}$. Define the variety $R_{d}$ of $k$-representations of $\Gamma$ of dimension vector $d$ by $R_{d}=$ $\bigoplus_{a: i \rightarrow j} \operatorname{Hom}\left(k^{d_{i}}, k^{d_{j}}\right)$. The group $G_{d}=\prod_{i \in I} \mathrm{GL}\left(k^{d_{i}}\right)$ acts on $R_{d}$ via $\left(g_{i}\right)\left(M_{a}\right)=$ $\left(g_{j} M_{a} g_{i}^{-1}\right)_{a: i \rightarrow j}$. By definition, the orbits of $G_{d}$ in $R_{d}$ correspond bijectively to the isomorphism classes of representations of $\Gamma$ of dimension vector $d$. Denote by $\mathcal{O}_{A}$ the $G_{d}$-orbit of a representation $A$. Since $R_{d}$ is irreducible and there are only finitely many orbits by Gabriel's theorem, $R_{d}$ contains a unique dense orbit. It is well known that (see, e.g., [3])

$$
\operatorname{codim} R_{d} \mathcal{O}_{A}=\operatorname{dim}_{\operatorname{Ext}_{\Gamma}^{1}}^{1}(A, A) .
$$

It follows that there exists a unique (up to isomorphism) representation $M^{+}$of dimension vector $d$ such that $\operatorname{Ext}_{\Gamma}^{1}\left(M^{+}, M^{+}\right)=0$. Since the $I$-graded $k$-space $V$ is uniquely determined by its signed dimension vector $\gamma-d$, uniqueness and existence follow.

As in [7, Proof of Theorem 1.10], Propositions 3.5 and 3.6 imply the following:

Theorem 3.7. The simplicial cones $\mathbf{R}_{\geq 0} C$ generated by all $\Gamma$-clusters $C$ form a smooth complete simplicial fan $\Delta_{\Gamma}$ in the ambient real vector space $Q_{\mathbf{R}}$ of the root system.

Remark 3.8. The "positive part" of $\Delta_{\Gamma}$ was studied by C. Riedtmann and A. Schofield [11] and L. Unger [15] following a suggestion of C. M. Ringel.

\section{EXTENDED REFLECTION FUNCTORS}

We recall the well-known reflection functors on quiver representations [1]. Let $i \in I$ be a source (resp. sink) in $\Gamma$, and let $s_{i} \Gamma$ be the quiver obtained by reversing all arrows pointing from (resp. to) $i$. Given a representation $M \in \operatorname{rep} \Gamma$, define a representation $S_{i}(M) \in \operatorname{rep} s_{i} \Gamma$ by

$$
S_{i}(M)_{i}=\left\{\begin{array}{ll}
\operatorname{Coker}\left(\bigoplus_{a: i \rightarrow j} M_{a}\right), & i \text { a source } \\
\operatorname{Ker}\left(\bigoplus_{a: j \rightarrow i} M_{a}\right), & i \text { a sink }
\end{array}\right\}, S_{i}(M)_{j}=M_{j} \text { for all } j \neq i,
$$

and the maps $S_{i}(M)_{a}$ are either unchanged, or derived in an obvious way from the canonical injection or projection maps. The functor $S_{i}$ is defined on morphisms using the canonical induced maps on cokernels (resp. kernels).

Let rep ${ }^{i} \Gamma$ denote the full subcategory of rep $\Gamma$ consisting of representations without $E_{i}$ as a direct summand. Then $S_{i}$ induces an equivalence of categories

$$
S_{i}: \operatorname{rep}{ }^{i} \Gamma \stackrel{\sim}{\rightarrow} \operatorname{rep}{ }^{i} s_{i} \Gamma .
$$

4.1. Definition and basic properties. Assume that $i \in I$ is a source or a sink in $\Gamma$. We will now define a reflection functor

$$
\Sigma_{i}: \operatorname{rep} \widetilde{\Gamma} \rightarrow \operatorname{rep} \widetilde{s_{i} \Gamma},
$$

which has the advantage - over the usual reflection functor $S_{i}$ - that it induces a bijection on isomorphism classes of decorated representations.

Let $M=M^{+} \oplus V$ be an object of rep $\widetilde{\Gamma}$. Suppose first that $i$ is a source in $\Gamma$, and consider the canonical exact sequence of $\Gamma$-representations

$$
0 \rightarrow E_{i} \otimes \operatorname{Hom}_{\Gamma}\left(E_{i}, M^{+}\right) \rightarrow M^{+} \rightarrow M^{\prime} \rightarrow 0 .
$$


Note for future reference the following well-known exact sequences:

$$
0 \rightarrow \operatorname{Hom}_{\Gamma}\left(E_{i}, M^{+}\right) \rightarrow M_{i} \rightarrow \bigoplus_{i \rightarrow j} M_{j} \rightarrow \operatorname{Ext}_{\Gamma}^{1}\left(E_{i}, M^{+}\right) \rightarrow 0 .
$$

The analogue for $i$ a sink is

$$
0 \rightarrow \operatorname{Hom}_{\Gamma}\left(M^{+}, E_{i}\right) \rightarrow M_{i}^{*} \rightarrow \bigoplus_{j \rightarrow i} M_{j}^{*} \rightarrow \operatorname{Ext}_{\Gamma}^{1}\left(M^{+}, E_{i}\right) \rightarrow 0
$$

Both these sequences are special cases of [12, Lemma 2.1].

In particular, (4.3) implies the natural isomorphism

$$
\operatorname{Hom}_{\Gamma}\left(E_{i}, M^{+}\right) \simeq \operatorname{Ker}\left(\bigoplus_{a: i \rightarrow j} M_{a}\right)
$$

Returning to (4.2), it is clear that $\operatorname{Hom}_{\Gamma}\left(E_{i}, M^{\prime}\right)=0$; thus $M^{\prime} \in \operatorname{rep}{ }^{i} \Gamma$. We define $\Sigma_{i}(M)=\bar{M}^{+} \oplus \bar{V}$ by setting

$$
\bar{M}^{+}=S_{i}\left(M^{\prime}\right) \oplus\left(E_{i} \otimes V_{i}\right), \quad \bar{V}=\left(E_{i}^{-} \otimes \operatorname{Hom}_{\Gamma}\left(E_{i}, M^{+}\right)\right) \oplus \bigoplus_{j \neq i}\left(E_{j}^{-} \otimes V_{j}\right)
$$

More explicitly, $\Sigma_{i}(M)$ replaces $M_{i}$ by $\operatorname{Coker}\left(\bigoplus_{a: i \rightarrow j} M_{a}\right) \oplus V_{i}$, replaces $V_{i}$ by $\operatorname{Ker}\left(\bigoplus_{a: i \rightarrow j} M_{a}\right)$, and leaves the rest of the spaces unchanged.

To define $\Sigma_{i}$ on morphisms, let $N=N^{+} \oplus W$ be another object of rep $\widetilde{\Gamma}$, and define $N^{\prime}, \bar{N}, \bar{W}$ in the same way as above. Let $f=f^{+} \oplus\left(\bigoplus_{j} \varphi_{j}\right)$ be a morphism from $M$ to $N$ in rep $\widetilde{\Gamma}$. Since $\operatorname{Hom}_{\Gamma}\left(E_{i}, N^{\prime}\right)=0$, the map $f^{+}$induces maps

$$
\psi: \operatorname{Hom}_{\Gamma}\left(E_{i}, M^{+}\right) \rightarrow \operatorname{Hom}_{\Gamma}\left(E_{i}, N^{+}\right), \quad f^{\prime}: M^{\prime} \rightarrow N^{\prime} .
$$

We then define the morphism $\Sigma_{i}(f)$ as the direct sum of the morphisms

$$
S_{i}\left(f^{\prime}\right) \oplus \varphi_{i}: \bar{M}^{+} \rightarrow \bar{N}^{+}
$$

and

$$
\psi \oplus \bigoplus_{j \neq i} \varphi_{j}: \bar{V} \rightarrow \bar{W}
$$

More explicitly, $\Sigma_{i}(f)$ replaces the map $f_{i}$ by $g \oplus \varphi_{i}$ (with respect to the above sum decomposition), where $g$ is the map induced by $f_{i}$ on the cokernels, replaces $\varphi_{i}$ by the map $h$ induced on the kernels, and leaves the rest of the maps unchanged.

To treat the case when $i$ is a sink, we use the duality functor $D$ (see Section 3). This allows us to reduce the case of a sink to that of a source by defining

$$
\Sigma_{i}=D \circ \Sigma_{i} \circ D \text {. }
$$

Explicitly, take the canonical exact sequence

$$
0 \rightarrow M^{\prime} \rightarrow M^{+} \rightarrow E_{i} \otimes \operatorname{Hom}_{\Gamma}\left(M^{+}, E_{i}\right)^{*} \rightarrow 0 .
$$

Then we have $\Sigma_{i}(M)=\bar{M}^{+} \oplus \bar{V}$, where

$$
\bar{M}^{+}=S_{i}\left(M^{\prime}\right) \oplus\left(E_{i} \otimes V_{i}\right)
$$

and

$$
\bar{V}=\left(E_{i}^{-} \otimes \operatorname{Hom}_{\Gamma}\left(M^{+}, E_{i}\right)^{*}\right) \oplus \bigoplus_{j \neq i}\left(E_{j}^{-} \otimes V_{j}\right) .
$$

The description of the action of $\Sigma_{i}$ on morphisms is similar to the case of a source and is left to the reader. 
The following proposition summarizes some simple properties of the extended reflection functors. All of them are immediate from the definitions.

\section{Proposition 4.1.}

(1) If $M \in \operatorname{rep}{ }^{i} \Gamma \subset \operatorname{rep} \widetilde{\Gamma}$, then $\Sigma_{i}(M)=S_{i}(M)$.

(2) We have $\Sigma_{i}\left(E_{i}\right)=E_{i}^{-}$and $\Sigma_{i}\left(E_{i}^{-}\right)=E_{i}$.

(3) We have $\Sigma_{i}\left(E_{j}^{-}\right)=E_{j}^{-}$whenever $i \neq j$.

(4) Suppose $i$ is a source (resp. a sink) in $\Gamma$, and $M \in \operatorname{rep}{ }^{i} \Gamma \subset \operatorname{rep} \widetilde{\Gamma}$. Let $f$ be any morphism from $M$ to $E_{i}$ (resp. from $E_{i}$ to $M$ ). Then $\Sigma_{i}(f)=0$.

Proposition 4.2. For any decorated representation $M \in \operatorname{rep} \widetilde{\Gamma}$ and any vertex $i \in I$ we have $\Sigma_{i}^{2}(M) \simeq M$.

Proof. Assume $i \in I$ to be a source in $\Gamma$ (the case of a sink follows by duality). For a decorated representation $M=M^{+} \oplus V \in \operatorname{rep} \widetilde{\Gamma}$, denote by $N=N^{+} \oplus W$ the decorated representation $\Sigma_{i}(M)$. (In the notation of 4.5 , we have $N=\bar{M}$ and $W=\bar{V}$.) By definition, we have

$$
\Sigma_{i}^{2}(M)=\bar{N}^{+} \oplus \bar{W} .
$$

Here

$$
\bar{N}^{+}=S_{i}\left(N^{\prime}\right) \oplus\left(E_{i} \otimes W_{i}\right) \simeq M^{\prime} \oplus\left(E_{i} \otimes \operatorname{Hom}_{\Gamma}\left(E_{i}, M^{+}\right)\right)
$$

and

$$
\begin{aligned}
\bar{W} & =\left(E_{i}^{-} \otimes \operatorname{Hom}_{s_{i} \Gamma}\left(S_{i}\left(N^{\prime}\right) \oplus\left(E_{i} \otimes V_{i}\right), E_{i}\right)\right)^{*} \oplus \bigoplus_{j \neq i}\left(E_{j}^{-} \otimes W_{j}\right) \\
& \simeq\left(E_{i}^{-} \otimes V_{i}\right) \oplus \bigoplus_{j \neq i}\left(E_{j}^{-} \otimes V_{j}\right) \simeq V .
\end{aligned}
$$

Note that $M^{\prime} \oplus\left(E_{i} \otimes \operatorname{Hom}_{\Gamma}\left(E_{i}, M^{+}\right)\right)$is (non-canonically) isomorphic to $M^{+}$since the sequence (4.2) splits. Therefore $\Sigma_{i}^{2}(M) \simeq M^{+} \oplus V=M$.

Corollary 4.3. The functor $\Sigma_{i}$ induces an isomorphism of Grothendieck groups $\Sigma_{i}: K_{0}(\operatorname{rep} \widetilde{\Gamma}) \stackrel{\sim}{\rightarrow} K_{0}\left(\operatorname{rep} \widetilde{s_{i} \Gamma}\right)$.

We define piecewise-linear reflections $\sigma_{i}$ on $Q$ as in [7, 2.2]. For the simply-laced case, this can be stated as follows:

Definition 4.4. For $i \in I$, define $\sigma_{i}: Q \rightarrow Q$ by

$$
\left[\sigma_{i} \alpha: \alpha_{j}\right]=\left\{\begin{array}{cl}
{\left[\alpha: \alpha_{j}\right],} & j \neq i \\
-\left[\alpha: \alpha_{i}\right]+\sum_{k-i} \max \left(\left[\alpha: \alpha_{k}\right], 0\right), & j=i
\end{array}\right.
$$

where $k-i$ means that $k$ and $i$ are joined by an edge in $I$.

Lemma 4.5. For any source or sink $i \in I$ of $\Gamma$, and any object $M=M^{+} \oplus V \in$ rep $\widetilde{\Gamma}$ such that $\operatorname{dim} M_{i} \cdot \operatorname{dim} V_{i}=0$, we have $\underline{\operatorname{sdim}}\left(\Sigma_{i}(M)\right)=\sigma_{i}(\underline{\operatorname{sdim}}(M))$.

Proof. Let $i$ be a source in $\Gamma$ (the case of a sink is dual). The reflection functor $\Sigma_{i}$ does not change any component $M_{j}$ or $V_{j}$ for $j \neq i$, which corresponds to the first part of the above definition of $\sigma_{i}$. So we concentrate on the $i$-components. By definition, we have $\left[\underline{\operatorname{sim}} M: \alpha_{i}\right]=\operatorname{dim} M_{i}-\operatorname{dim} V_{i}$. Using the definition of $\Sigma_{i}$, we compute:

$$
\left[\underline{\operatorname{sdim}} \Sigma_{i}(M): \alpha_{i}\right]=\operatorname{dim} S_{i}\left(M^{\prime}\right)_{i}+\operatorname{dim} V_{i}-\operatorname{dim} \operatorname{Hom}_{\Gamma}\left(E_{i}, M\right) .
$$


In view of the exact sequence (4.3),

$$
\operatorname{dim} S_{i}\left(M^{\prime}\right)_{i}-\operatorname{dim} \operatorname{Hom}_{\Gamma}\left(E_{i}, M\right)=-\operatorname{dim} M_{i}+\sum_{i \rightarrow j} \operatorname{dim} M_{j} .
$$

Therefore,

$$
\begin{aligned}
{\left[\underline{\operatorname{sdim}} \Sigma_{i}(M): \alpha_{i}\right] } & =-\operatorname{dim} M_{i}+\sum_{i \rightarrow j} \operatorname{dim} M_{j}+\operatorname{dim} V_{i} \\
& =-\left[\underline{\operatorname{sdim}} M: \alpha_{i}\right]+\sum_{i \rightarrow j} \operatorname{dim} M_{j} .
\end{aligned}
$$

Under the assumption $\operatorname{dim} M_{i} \cdot \operatorname{dim} V_{i}=0$, this last expression is the same as in Definition 4.4 .

Corollary 4.6. For all roots $\alpha \in \Phi_{\geq-1}$, and any $i \in I$ that is a source or a sink in $\Gamma$, we have

$$
\underline{\operatorname{sdim}}\left(\sum_{i}\left(U_{\alpha}^{\Gamma}\right)\right)=\sigma_{i}\left(\underline{\operatorname{sdim}}\left(U_{\alpha}^{\Gamma}\right)\right) .
$$

Proof. Notice that for any indecomposable object $M \in \operatorname{rep} \widetilde{\Gamma}$, we have $\operatorname{dim} M_{i}$. $\operatorname{dim} V_{i}=0$ for all $i \in I$.

4.2. Extended reflection functors and compatibility degree. Recall the bifunctor $E_{\Gamma}$ on decorated representations introduced in (3.1).

Theorem 4.7. If $i \in I$ is a source or a sink in $\Gamma$, then $E_{s_{i} \Gamma}\left(\Sigma_{i}(M), \Sigma_{i}(N)\right) \simeq$ $E_{\Gamma}(M, N)$ for any decorated representations $M$ and $N$.

Proof. We start with a lemma which is an immediate consequence of (4.3) and (4.4).

Lemma 4.8. Let $A$ be a representation of $\Gamma$. If $i$ is a source in $\Gamma$, then

$$
S_{i}(A)_{i} \simeq \operatorname{Ext}_{\Gamma}^{1}\left(E_{i}, A\right) .
$$

If $i$ is a sink in $\Gamma$, then

$$
S_{i}(A)_{i} \simeq \operatorname{Ext}_{\Gamma}^{1}\left(A, E_{i}\right)^{*} .
$$

Returning to the proof of Theorem 4.7, we will only treat the case of a source $i \in I$; the case of a sink is dual. As usual, we write $M=M^{+} \oplus V$ and $N=N^{+} \oplus W$. Recall the (non-canonical) decompositions

$$
M^{+}=M^{\prime} \oplus\left(E_{i} \otimes X\right), \quad N^{+}=N^{\prime} \oplus\left(E_{i} \otimes Y\right),
$$

where $X=\operatorname{Hom}_{\Gamma}\left(E_{i}, M^{+}\right)$and $Y=\operatorname{Hom}_{\Gamma}\left(E_{i}, N^{+}\right)$. Using the definition of $\Sigma_{i}$, we decompose $E_{s_{i} \Gamma}\left(\Sigma_{i}(M), \Sigma_{i}(N)\right)$

$$
\begin{aligned}
= & \operatorname{Ext}_{s_{i} \Gamma}^{1}\left(S_{i}\left(M^{\prime}\right), S_{i}\left(N^{\prime}\right)\right) \oplus\left(\operatorname{Ext}_{s_{i} \Gamma}^{1}\left(S_{i}\left(M^{\prime}\right), E_{i}\right) \otimes W_{i}\right) \\
& \oplus \operatorname{Ext}_{s_{i} \Gamma}^{1}\left(S_{i}\left(N^{\prime}\right), S_{i}\left(M^{\prime}\right)\right)^{*} \oplus\left(\operatorname{Ext}_{s_{i} \Gamma}^{1}\left(S_{i}\left(N^{\prime}\right), E_{i}\right) \otimes V_{i}\right)^{*} \\
& \oplus \operatorname{Hom}\left(S_{i}\left(M^{\prime}\right)_{i}, Y\right) \oplus \operatorname{Hom}\left(V_{i}, Y\right) \oplus \bigoplus_{j \neq i} \operatorname{Hom}\left(M_{j}, W_{j}\right) \\
& \oplus \operatorname{Hom}\left(X, S_{i}\left(N^{\prime}\right)_{i}\right) \oplus \operatorname{Hom}\left(X, W_{i}\right) \oplus \bigoplus_{j \neq i} \operatorname{Hom}\left(V_{j}, N_{j}\right) .
\end{aligned}
$$


Since the classical reflection functor $S_{i}$ induces an equivalence of categories rep ${ }^{i} \Gamma \stackrel{\sim}{\rightarrow}$ rep ${ }^{i} s_{i} \Gamma$, we have

$$
\begin{aligned}
& \operatorname{Ext}_{s_{i} \Gamma}^{1}\left(S_{i}\left(M^{\prime}\right), S_{i}\left(N^{\prime}\right)\right) \simeq \operatorname{Ext}_{\Gamma}^{1}\left(M^{\prime}, N^{\prime}\right), \\
& \operatorname{Ext}_{s_{i} \Gamma}^{1}\left(S_{i}\left(N^{\prime}\right), S_{i}\left(M^{\prime}\right)\right)^{*} \simeq \operatorname{Ext}_{\Gamma}^{1}\left(N^{\prime}, M^{\prime}\right)^{*} .
\end{aligned}
$$

Furthermore, applying the second part of Lemma 4.8 to $A=S_{i}\left(M^{\prime}\right)$ and using Proposition 4.2, we obtain

$$
\operatorname{Ext}_{s_{i} \Gamma}^{1}\left(S_{i}\left(M^{\prime}\right), E_{i}\right) \otimes W_{i} \simeq \operatorname{Hom}\left(M_{i}^{\prime}, W_{i}\right) .
$$

Similarly,

$$
\left(\operatorname{Ext}_{s_{i} \Gamma}^{1}\left(S_{i}\left(N^{\prime}\right), E_{i}\right) \otimes V_{i}\right)^{*} \simeq \operatorname{Hom}\left(V_{i}, N_{i}^{\prime}\right) .
$$

Substituting the expressions provided by (4.6) - (4.9) into the above decomposition of $E_{s_{i} \Gamma}\left(\Sigma_{i}(M), \Sigma_{i}(N)\right)$ and regrouping the terms, we can write it as the direct sum of the following four terms:

$$
\begin{aligned}
& \left(\operatorname{Ext}_{\Gamma}^{1}\left(M^{\prime}, N^{\prime}\right) \oplus \operatorname{Hom}\left(X, S_{i}\left(N^{\prime}\right)_{i}\right)\right) \\
& \oplus\left(\operatorname{Ext}_{\Gamma}^{1}\left(N^{\prime}, M^{\prime}\right)^{*} \oplus \operatorname{Hom}\left(S_{i}\left(M^{\prime}\right)_{i}, Y\right)\right) \\
& \oplus\left(\operatorname{Hom}\left(M_{i}^{\prime}, W_{i}\right) \oplus \bigoplus_{j \neq i} \operatorname{Hom}\left(M_{j}, W_{j}\right) \oplus \operatorname{Hom}\left(X, W_{i}\right)\right) \\
& \oplus\left(\operatorname{Hom}\left(V_{i}, N_{i}^{\prime}\right) \oplus \operatorname{Hom}\left(V_{i}, Y\right) \oplus \bigoplus_{j \neq i} \operatorname{Hom}\left(V_{j}, N_{j}\right)\right) .
\end{aligned}
$$

To complete the proof, we show that these four terms can be identified with the four terms in (3.1) in the same order.

First we have

$$
\begin{aligned}
\operatorname{Ext}_{\Gamma}^{1}\left(M^{+}, N^{+}\right) & =\operatorname{Ext}_{\Gamma}^{1}\left(M^{\prime} \oplus\left(E_{i} \otimes X\right), N^{\prime} \oplus\left(E_{i} \otimes Y\right)\right) \\
& =\operatorname{Ext}_{\Gamma}^{1}\left(M^{\prime}, N^{\prime}\right) \oplus\left(\operatorname{Ext}_{\Gamma}^{1}\left(E_{i}, N^{\prime}\right) \otimes X^{*}\right) .
\end{aligned}
$$

This agrees with (4.10) by Lemma 4.8. The identification of $\operatorname{Ext}_{\Gamma}^{1}\left(N^{+}, M^{+}\right)^{*}$ with (4.11) is proved dually. Finally, the terms in (4.12) and (4.13) obviously equal the last two terms in (3.1).

Remark 4.9. The isomorphism given in the theorem is not natural, since the $\Sigma_{i}$ do not provide an equivalence of categories. But it can be shown (with the same proof as above) that there exists an isomorphism of functors $E_{s_{i} \Gamma}\left(\Sigma_{i}, \Sigma_{i}\right) \simeq E_{\Gamma}\left(\Sigma_{i}^{2}, \Sigma_{i}^{2}\right)$.

As an immediate consequence of the definition (3.3), Corollary 4.6 and Theorem 4.7, we get:

Proposition 4.10. If $i \in I$ is a source or a sink in $\Gamma$, then $\left(\sigma_{i} \alpha \| \sigma_{i} \beta\right)_{s_{i} \Gamma}=(\alpha \| \beta)_{\Gamma}$ for all $\alpha, \beta \in \Phi_{\geq-1}$.

This result has the following implications.

Corollary 4.11. If $\Gamma$ and $\Gamma^{\prime}$ are two quivers with the same underlying Dynkin graph, then the simplicial complexes $\Delta_{\Gamma}$ and $\Delta_{\Gamma^{\prime}}$ are isomorphic to each other.

Proof. If $\Gamma^{\prime}=s_{i} \Gamma$ for some source or sink $i$, then by Proposition 4.10, the permutation $\sigma_{i}$ of $\Phi_{\geq-1}$ transforms $\Delta_{\Gamma}$ to $\Delta_{\Gamma^{\prime}}$. Any two quivers can be obtained from each other by a sequence of such transformations (see, e.g., [1, Theorem 1.2]). 
We now show that the notion of $\Gamma$-compatibility degree includes as a special case the compatibility degree introduced in [7, Section 3.1]. Let $\Gamma_{0}$ be an alternating quiver (i.e., such that each vertex is a source or a sink). Denote the set of sources of $\Gamma_{0}$ by $I^{+}$, and the set of sinks by $I^{-}$. Following [7], we define the transformations $\tau_{+}$and $\tau_{-}$by

$$
\tau_{+}=\prod_{i \in I^{+}} \sigma_{i}, \quad \tau_{-}=\prod_{i \in I^{-}} \sigma_{i}
$$

Recall from [7] that the compatibility degree is characterized by the following two properties:

$$
\begin{aligned}
& \left(-\alpha_{i} \| \beta\right)=\max \left(\left[\beta: \alpha_{i}\right], 0\right) \text { for all } i \in I \text { and } \beta \in \Phi_{\geq-1} \\
& \left(\tau_{\epsilon} \alpha \| \tau_{\epsilon} \beta\right)=(\alpha \| \beta) \text { for } \epsilon \in\{+,-\} .
\end{aligned}
$$

Corollary 4.12. The $\Gamma_{0}$-compatibility degree coincides with the compatibility degree defined by (4.14)-(4.15). Therefore, $\Gamma_{0}$-clusters coincide with the clusters in the sense of $[7]$.

Proof. It suffices to show that the $\Gamma_{0}$-compatibility degree satisfies (4.14)-(4.15). The property (4.14) follows at once from (3.3). For (4.15), notice that $\tau_{+}$and $\tau_{-}$ send $\Gamma_{0}$ to $\Gamma_{0}^{\text {op }}$, and apply Propositions 4.10 and 3.1 .

\section{The Reflection Groupoid}

In this section, we show that the group generated by the two involutions $\tau_{+}, \tau_{-}$ has a natural interpretation in the context of decorated quiver representations. To do this, we consider the following more general setup. Suppose that $I$ is an arbitrary finite tree. We associate to $I$ two groupoids defined as follows.

Let $R_{0}(I)$ denote the groupoid whose objects are given by the set $\operatorname{Quiv}(I)$ of all quivers obtained by specifying an orientation of $I$, and whose morphisms are defined as follows. First of all, for any $\Gamma \in \operatorname{Quiv}(I)$ and any source or sink $i$ in $\Gamma$, there is an "elementary" isomorphism $\Sigma_{i}: \Gamma \stackrel{\sim}{\rightarrow} s_{i} \Gamma$. The morphisms in $R_{0}(I)$ are arbitrary compositions of these isomorphisms, subject to the following relations (where 1 denotes the appropriate identity automorphism):

(R1) $\Sigma_{i}^{2}=1$,

(R2) $\Sigma_{i} \Sigma_{j}=\Sigma_{j} \Sigma_{i}$ whenever $i$ and $j$ are not linked in $I$.

In both cases, the relation holds whenever the maps and their compositions are defined.

We also consider another groupoid $R(I)$ with the same set of objects Quiv $(I)$, obtained from $R_{0}(I)$ by adjoining one more "elementary isomorphism" $D: \Gamma \stackrel{\sim}{\rightarrow} \Gamma^{\mathrm{op}}$, for any $\Gamma \in \operatorname{Quiv}(I)$, satisfying the following relations (as before, they must hold whenever the morphisms and compositions on both sides are defined):

(R3) $D^{2}=1$,

(R4) $D \Sigma_{i}=\Sigma_{i} D$ for all $i \in I$.

Let $I=I^{+} \cup I^{-}$be a disjoint union of $I$ into two completely disconnected subsets, i.e., no two vertices in $I^{+}$(respectively, $I^{-}$) have a common edge. Such a decomposition exists because $I$ is a tree, and is unique up to interchanging $I^{+}$and $I^{-}$.

Denote by $\Gamma_{0}$ the alternating orientation of $I$ in which every element of $I^{+}$is a source. Let $\Sigma_{+}=\prod_{i \in I^{+}} \Sigma_{i}$ and $\Sigma_{-}=\prod_{i \in I^{-}} \Sigma_{i}$. Note that, by the relation (R2), these morphisms do not depend on the order in which the compositions are taken. 
We can regard $\Sigma_{+}, \Sigma_{-}$as morphisms (in either of the groupoids $R_{0}(I)$ or $R(I)$ ) from $\Gamma_{0}$ to the opposite orientation $\Gamma_{0}^{\mathrm{op}}$, or from $\Gamma_{0}^{\mathrm{op}}$ to $\Gamma_{0}$. The main result of this section can now be stated as follows:

Theorem 5.1. (a) The automorphism group $\operatorname{Aut}_{R_{0}(I)}\left(\Gamma_{0}\right)$ is generated by $\Sigma_{+} \Sigma_{-}$ and $\Sigma_{-} \Sigma_{+}$.

(b) The automorphism group $\operatorname{Aut}_{R(I)}\left(\Gamma_{0}\right)$ is generated by $D \Sigma_{+}$and $D \Sigma_{-}$.

Before embarking on the proof of this theorem, we note the following:

Remark 5.2. Let $T_{+}=D \Sigma_{+}: \Gamma_{0} \stackrel{\sim}{\rightarrow} \Gamma_{0}$ and let $T_{-}=D \Sigma_{-}: \Gamma_{0} \stackrel{\sim}{\rightarrow} \Gamma_{0}$. Theorem 5.1 states that $T_{+}$and $T_{-}$generate the automorphism group $\operatorname{Aut}_{R(I)}\left(\Gamma_{0}\right)$. In the case where $I$ is a simply-laced Dynkin diagram, we can interpret the isomorphisms $\Sigma_{i}$ in $R(I)$ as the extended reflection functors of Section 4 and the isomorphism $D$ as the usual duality rep $\widetilde{\Gamma} \stackrel{\sim}{\rightarrow}$ rep $\widetilde{\Gamma^{\text {op }}}$. Using Corollary 4.6 and the fact that $\underline{\operatorname{sdim}}(D M)=\underline{\operatorname{sdim}}(M)$ for all objects $M$ of rep $\widetilde{\Gamma}$ (for any $\Gamma$ ), we see that if $T_{+}$ and $T_{-}$are regarded as bijections of the set of objects of rep $\widetilde{\Gamma}_{0}$, they induce the involutions $\tau_{+}$and $\tau_{-}$of the root lattice $Q$ introduced in [7].

Theorem 5.1 has the following corollary:

Corollary 5.3. Let $\Gamma$ be any element of $\operatorname{Quiv}(I)$. Then:

(a) The automorphism group $\operatorname{Aut}_{R_{0}(I)}(\Gamma)$ is cyclic.

(b) The automorphism group $\operatorname{Aut}_{R(I)}(\Gamma)$ is generated by two involutions.

Proof. This follows immediately from Theorem 5.1, using the fact that $\operatorname{Quiv}(I)$ is connected under the operations $s_{i}$ (by [1, Theorem 1.2]), and so all automorphism groups in the groupoid are conjugate.

Proof of Theorem 5.1. We note first of all that (b) is an easy formal consequence of (a): an element of $\operatorname{Aut}_{R_{0}(I)}\left(\Gamma_{0}\right)$ is either $\left(\Sigma_{+} \Sigma_{-}\right)^{k}=\left(D \Sigma_{+} D \Sigma_{-}\right)^{k}$, or $\left(\Sigma_{-} \Sigma_{+}\right)^{k}=$ $\left(D \Sigma_{-} D \Sigma_{+}\right)^{k}$; and an element of $\operatorname{Aut}_{R(I)}\left(\Gamma_{0}\right) \backslash \operatorname{Aut}_{R_{0}(I)}\left(\Gamma_{0}\right)$ can be written as $D \Sigma_{+} \Sigma^{\prime}$ with $\Sigma^{\prime} \in \operatorname{Aut}_{R_{0}(I)}\left(\Gamma_{0}\right)$.

Turning to (a), let $\Sigma$ be any morphism in $R_{0}(I)$. By analogy with Coxeter groups, we define the length of $\Sigma$, denoted $\ell(\Sigma)$, to be the minimum length of an expression $\Sigma=\Sigma_{i_{1}} \Sigma_{i_{2}} \cdots \Sigma_{i_{r}}$. We call an expression of minimal length for $\Sigma$ a reduced expression for $\Sigma$. In the sequel, we will often write $i$ instead of $\Sigma_{i}$ to simplify the notation.

The following Lemma is an immediate consequence of the relations (R1) and (R2).

Lemma 5.4. An expression $\Sigma_{i_{1}} \Sigma_{i_{2}} \cdots \Sigma_{i_{r}}$ is reduced if and only if between any two occurrences of the same $i$ there is an occurrence of $j$ for some $j$ linked to $i$.

We can strengthen this as follows:

Lemma 5.5. An expression $\Sigma_{i_{1}} \Sigma_{i_{2}} \cdots \Sigma_{i_{r}}$ for a morphism in $R_{0}(I)$ is reduced if and only if between any two occurrences of $i$ there is precisely one occurrence of $j$ for each $j$ linked to $i$.

Proof. The "if" part follows from Lemma 5.4; so we only have to show the "only if" statement. So suppose that $\Sigma_{i_{1}} \Sigma_{i_{2}} \cdots \Sigma_{i_{r}}$ is a reduced expression. We argue by induction on the distance between two consecutive occurrences of $i$. If the distance 
is 1 , then the corresponding interval is $\Sigma_{i} \Sigma_{j} \Sigma_{i}$. Since this expression is reduced, $j$ is linked to $i$, and in order for the second $\Sigma_{i}$ to be applicable, $j$ must be the only element of $I$ that is linked to $i$; so we are done.

For the inductive step, we must first show that every $j$ linked to $i$ appears at most once between two consecutive occurrences of $i$. This is true since, by induction, if we had two occurrences of some $j$ linked to $i$, between them $i$ should occur, a contradiction. Then notice that all $j$ linked to $i$ must appear between the two consecutive occurrences of $i$, because we have to reverse all arrows at $i$.

Lemma 5.6. Suppose that $\Sigma_{i_{1}} \Sigma_{i_{2}} \cdots \Sigma_{i_{p}}$ is a reduced expression for a morphism in $R_{0}(I)$ that starts at an arbitrary orientation of $I$ and ends up at $\Gamma_{0}$ or $\Gamma_{0}^{o p}$. Then, for every $i$ that appears in the reduced expression, we have precisely one of the following:

(i) no $j$ linked to $i$ appears before the first occurrence of $i$, or

(ii) every $j$ linked to $i$ appears exactly once before the first occurrence of $i$.

Proof. This follows easily from Lemma 5.5. Let $\Sigma^{\prime}=\Sigma_{i} \Sigma$; note that this composition is well-defined since $\Sigma$ ends up at $\Gamma_{0}$ or $\Gamma_{0}^{\text {op }}$, from which every $\Sigma_{i}$ is applicable. If $\Sigma_{i} \Sigma_{i_{1}} \Sigma_{i_{2}} \cdots \Sigma_{i_{p}}$ is reduced, then (ii) holds by Lemma 5.5 ; otherwise (i) holds by Lemma 5.4.

Lemma 5.7. Suppose that $\Sigma$ is a morphism of $R_{0}(I)$ that begins and ends at the same orientation $\Gamma$, and that $\Sigma$ is not the identity morphism. Then every reduced expression for $\Sigma$ contains all $i \in I$.

Proof. It is enough to show that if $i$ occurs in a reduced expression for $\Sigma$ and $j$ is linked to $i$, then $j$ also occurs. Suppose this is not the case. Then $i$ must occur at least twice, because the arrow linking $i$ and $j$ must be reversed at least twice by $\Sigma$. So $j$ must occur by Lemma 5.5.

Now everything is ready for the proof of Theorem 5.1(a). We need to show that every morphism $\Sigma$ in $R_{0}(I)$ that begins and ends at the same quiver $\Gamma$, where $\Gamma$ is $\Gamma_{0}$ or $\Gamma_{0}^{\mathrm{op}}$, has a reduced expression of the form $\left(\Sigma_{+} \Sigma_{-}\right)^{k}$ or $\left(\Sigma_{-} \Sigma_{+}\right)^{k}$ for some $k \in \mathbb{Z}_{\geq 0}$.

We proceed by induction on $\ell(\Sigma)$. There is nothing to prove when $\ell(\Sigma)=0$. So let us assume that $\Sigma$ is nontrivial, and that the statement is known for morphisms of smaller length. Fix a reduced expression for $\Sigma$. By Lemma 5.7, all $i \in I$ occur. By Lemma 5.6, $I$ is the disjoint union of two subsets: those satisfying 5.6(i) and those satisfying 5.6(ii). By definition, if $i$ and $j$ are linked, then they belong to different subsets, since whichever of $i$ and $j$ appears first in $\Sigma$ satisfies (i), while the other satisfies (ii). It follows that one of these parts is $I^{+}$, and the other is $I^{-}$. Suppose that $I^{+}$is the set of indices satisfying (i) (the argument if $I^{-}$is this set is similar). Commuting the first occurrences of $\Sigma_{i}$ for each $i \in I^{+}$to the beginning of our reduced expression, we obtain a reduced expression for $\Sigma$ of the form $\Sigma_{+} \Sigma^{\prime}$ with $\ell\left(\Sigma^{\prime}\right)=\ell(\Sigma)-\left|I^{+}\right|$, such that every reduced expression for $\Sigma^{\prime}$ starts with $\Sigma_{j}$ for some $j \in I^{-}$. Note that $\Sigma^{\prime}$ is a morphism from $\Gamma$ to $\Gamma^{\mathrm{op}}$.

Next consider the morphism $\Sigma^{\prime \prime}=\Sigma^{\prime} \Sigma_{+}$from $\Gamma^{\mathrm{op}}$ to $\Gamma^{\mathrm{op}}$. It is clear that $\ell\left(\Sigma^{\prime \prime}\right) \leq$ $\ell\left(\Sigma^{\prime}\right)+\left|I^{+}\right|=\ell(\Sigma)$. If $\ell\left(\Sigma^{\prime \prime}\right)<\ell(\Sigma)$, then by induction $\Sigma^{\prime \prime}$ has an expression $\left(\Sigma_{+} \Sigma_{-}\right)^{k}$ or $\left(\Sigma_{-} \Sigma_{+}\right)^{k}$, which implies the same claim for $\Sigma=\Sigma_{+} \Sigma^{\prime}=\Sigma_{+} \Sigma^{\prime \prime} \Sigma_{+}$.

So let us assume that $\ell\left(\Sigma^{\prime \prime}\right)=\ell(\Sigma)$ and take a reduced expression for $\Sigma^{\prime \prime}$ obtained by taking the original reduced expression for $\Sigma^{\prime}$ (recall that it starts with some 
$j \in I^{-}$) followed by any reduced expression for $\Sigma_{+}$. Applying to this expression the same argument as before, we conclude that by moving some $\Sigma_{j}$ 's for $j \in I^{-}$ to the left we obtain a reduced expression for $\Sigma^{\prime}$ that starts with $\Sigma_{-}$. This leads to a reduced expression for $\Sigma$ that starts with $\Sigma_{+} \Sigma_{-}$; by induction the rest of the reduced expression can be written in the desired form, and Theorem 5.1(a) follows.

\section{An APPLICATION TO QUiVER REPRESENTATIONS}

As before, let $\Gamma$ be a quiver whose underlying graph $I$ is a Dynkin diagram of the $A D E$-type. We say that a set $C$ of isomorphism classes of indecomposable representations of $\Gamma$ is Ext-free (also called a partial tilting set) if $\operatorname{Ext}^{1}(M, N)=0$ for every $M, N \in C$. For any nonnegative integer $k$, let $f_{\Gamma}^{+}(k)$ denote the number of these Ext-free sets of cardinality $k$.

Proposition 6.1. For every $k$, the number $f_{\Gamma}^{+}(k)$ depends only on the underlying Dynkin graph, not on the choice of an orientation $\Gamma$.

Proof. Using Gabriel's theorem, we identify isoclasses of indecomposable representations of $\Gamma$ with positive roots in $\Phi$. Under this identification, the Ext-free sets become positive $\Gamma$-compatible subsets of $\Phi_{\geq-1}$, in the terminology of Definition 3.2.

For every subset $J \subset I$, let $f_{\Gamma}(k, J)$ (resp. $f_{\Gamma}^{+}(k, J)$ ) denote the number of $\left.\Gamma\right|_{J}$-compatible subsets of cardinality $k$ in $\Phi(J)_{\geq-1}\left(\operatorname{resp} . \Phi(J)_{>0}\right)$; here $\left.\Gamma\right|_{J}$ is the orientation induced by $\Gamma$ on the full subgraph $J$ of $I$. Thus, we have, in particular, $f_{\Gamma}^{+}(k, I)=f_{\Gamma}^{+}(k)$. In view of Proposition 3.4, these numbers satisfy the following relations:

$$
f_{\Gamma}(k, J)=\sum_{K \subset J,|K| \leq k} f_{\Gamma}^{+}(k-|K|, J-K) .
$$

It follows that the numbers $f_{\Gamma}^{+}(k, J)$ can be expressed as integer linear combinations of the numbers $f_{\Gamma}(l, K)$ by using the Möbius inversion on the partially ordered set of pairs $(k, J)$ with the partial order given by

$$
(l, K) \leq(k, J) \Leftrightarrow K \subset J, k-l=|J-K| .
$$

Since this poset structure is independent of $\Gamma$, it remains to show that so are the numbers $f_{\Gamma}(k, I)$. But the latter statement is an immediate consequence of Corollary 4.11 .

For every $\Gamma$, the corresponding Ext-free sets form a simplicial complex, which was studied by C. Riedtmann and A. Schofield [11] and L. Unger [15] (see Remark 3.8). Proposition 6.1 shows that the $f$-vector of this complex depends only on the underlying Dynkin graph. Despite this fact, the complexes corresponding to different orientations of the same graph may be not isomorphic to each other.

Example 6.2. Let $I$ be of type $A_{3}$, and let $\Gamma_{0}$ (resp. $\Gamma_{1}$ ) be an alternating (resp. equi-oriented) orientation of $I$. The corresponding simplicial complexes are shown in Figure 1, and they are clearly non-isomorphic (in the figure, the vertices labeled by $i, i j$, and $i j k$ represent roots $\alpha_{i}, \alpha_{i}+\alpha_{j}$, and $\alpha_{i}+\alpha_{j}+\alpha_{k}$, respectively).

A recursive way to compute the numbers $f_{\Gamma}(k, I)$ is given by [7, Proposition 3.7]. The numbers $f_{\Gamma}^{+}(k, I)$ can then be found by the Möbius inversion described in the 

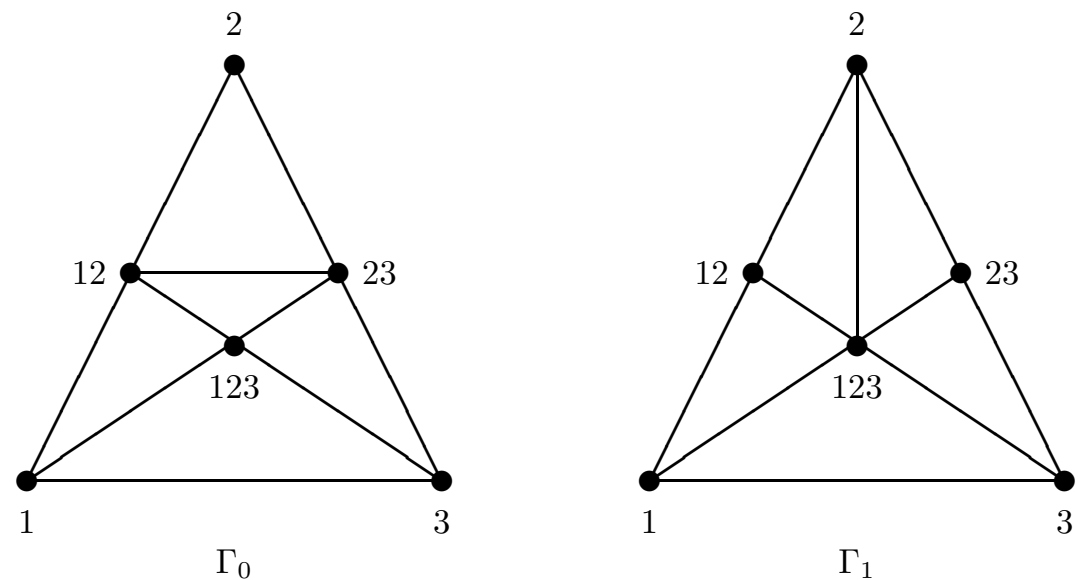

Figure 1. "Positive" simplicial complexes in type $A_{3}$

proof of Proposition 6.1. In particular, when $\Phi$ is irreducible, the total number $f_{\Gamma}^{+}(|I|, I)$ of positive $\Gamma$-clusters is given by $[7,(3.8)]$ :

$$
f_{\Gamma}^{+}(|I|, I)=\prod_{i \in I} \frac{e_{i}+h-1}{e_{i}+1},
$$

where the $e_{i}$ are the exponents of $\Phi$, and $h$ is the Coxeter number. It would be interesting to explain this formula using the theory of quiver representations.

\section{ACKNOWLEDGMENTS}

This work started in July 2001 when M. R. and A. Z. visited the University of Leicester, whose hospitality and financial support are gratefully acknowledged. A. Z. also thanks the Isaac Newton Institute for Mathematical Sciences in Cambridge, UK for supporting his travel to Leicester. M. R. thanks Northeastern University for kind hospitality and financial support during his visit in March 2002. Finally, M. R. and A. Z. thank the organizers of the meeting "Enveloping algebras and Algebraic Lie Representations" in April 28-May 4, 2002, as well as the staff at the Mathematisches Forschungsinstitut Oberwolfach, for providing an inspiring atmosphere and the opportunity to work together on the finishing stages of this project.

\section{REFERENCES}

[1] I. N. Bernstein, I. M. Gelfand and V. A. Ponomarev, Coxeter functors, and Gabriel's theorem, Uspehi Mat. Nauk 28 (1973), no. 2(170), 19-33. MR52:13876

[2] K. Bongartz, Tilted algebras, Representations of algebras (Puebla, 1980), 26-38, Lecture Notes in Math. 903, Springer-Verlag, Berlin and New York, 1981. MR83g:16053

[3] K. Bongartz, On degenerations and extensions of finite-dimensional modules, Adv. Math. 121 (1996), no. 2, 245-287. MR98e:16012

[4] R. Bott and C. Taubes, On the self-linking of knots, Topology and Physics, J. Math. Phys. 35 (1994), no. 10, 5247-5287. MR95g:57008

[5] F. Chapoton, S. Fomin and A. Zelevinsky, Polytopal realizations of generalized associahedra, Preprint math.CO/0202004, Canadian Math. Bulletin 45 (2002), no. 4, 537-566. 
[6] S. Fomin and A. Zelevinsky, Cluster algebras I: Foundations, J. Amer. Math. Soc. 15 (2002), 497-529.

[7] S. Fomin and A. Zelevinsky, $Y$-systems and generalized associahedra, Preprint hep-th/ 0111053, to appear in Ann. of Math. (2).

[8] D. Happel and C. M. Ringel, Tilted algebras, Trans. Amer. Math. Soc. 274 (1982), no. 2, 399-443. MR84d:16027

[9] A. Hubery, Quiver representations respecting a quiver automorphism: A generalisation of a theorem of Kac. Preprint math.RT/0203195.

[10] G. Lusztig, Introduction to quantum groups, Progress in Mathematics 110, Birkhäuser, Boston, MA, 1993. MR94m:17016

[11] C. Riedtmann and A. Schofield, On a simplicial complex associated with tilting modules, Comment. Math. Helv. 66 (1991), no. 1, 70-78. MR92a:16019

[12] C. M. Ringel, Representations of $K$-species and bimodules, J. Algebra 41 (1976), 269-302. MR54:10340

[13] J. D. Stasheff, Homotopy associativity of $H$-spaces. I, II, Trans. Amer. Math. Soc. 108 (1963), 275-292, 293-312. MR28:1623

[14] T. Tanisaki, Foldings of root systems and Gabriel's theorem, Tsukuba J. Math. 4 (1980), no. 1, 89-97. MR82g:16031

[15] L. Unger, The simplicial complex of tilting modules over quiver algebras, Proc. London Math. Soc. (3) 73 (1996), 27-46. MR97g:16019

Department of Mathematics and Computer Science, University of Leicester, UniverSITy RoAd, LEICESTER LE1 7RH, ENGLAND

BuGH Wuppertal, Gaussstrasse 20, D-42097 Wuppertal, Germany

Email address: reineke@math.uni-wuppertal.de

Department of Mathematics, Northeastern University, Boston, Massachusetts 02115

Email address: andrei@neu.edu 